\title{
Um retrato sobre o ensino do Pensamento Computacional em anos finais do Ensino Fundamental no Sertão Paraibano
}

\author{
Francisco Ribeiro dos Santos Júnior, CI - UFPB, ribeiro.juniorsantos@gmail.com \\ Danielle Rousy Dias Ricarte, CI - UFPB, danielle@ci.ufpb.br
}

\begin{abstract}
Resumo: O Pensamento Computacional, tido como uma das competências para habilidades exigidas no século XXI para o exercício da cidadania, está relacionada a capacidade de utilizar conceitos de computação para a resolução de problemas de diversas áreas de conhecimento. Ele abre caminhos para os futuros profissionais e ajuda no desenvolvimento mais efetivo de conhecimentos da capacidade de resolver problemas, apoiando e relacionando-se com outras ciências. Nesta perspectiva, o presente trabalho propôs investigar, através de uma pesquisa exploratória, o ensino de conceitos e abordagens do pensamento computacional nos anos finais do ensino fundamental ( $6^{\circ}$ ao $9^{\circ}$ ano), tendo como campo de pesquisa, escolas públicas do Sertão da Paraíba. Utilizou-se um questionário para coleta de informações a respeito do perfil socioprofissional dos professores e seu entendimento a respeito do Pensamento Computacional. Pode-se concluir que a definição de Pensamento Computacional ainda é desconhecida pela maioria dos participantes da pesquisa, além de ser amplamente associado ao uso de computadores e outras tecnologias digitais em sala de aula. Os conceitos de paralelização, análise de dados e coleta de dados, ligados a computação, foram identificados os mais abordados nas aulas, sendo matemática a disciplina que utiliza com maior frequência os conceitos abordados.
\end{abstract}

Palavras-chave: Educação Básica, Ensino de Computação, Pensamento Computacional, Resolução de Problemas.

Abstract: Computational Thinking (CP), considered one of the skills competencies required in the 21 st century for the exercise of citizenship, is related to the ability to use computing concepts to solve the most diverse problems in various areas of knowledge. Its application in Basic Education demands importance, since it opens paths for future professionals and helps in more effective development of knowledge about the ability to solve problems, supporting and relating to other science fields. In this perspective, the present work proposes to investigate, through an exploratory research, the teaching of concepts and approaches of computational thinking in the final years of elementary school (6th to 9th grade), having as a research field, public schools in the Sertão da Paraíba. Use a questionnaire to collect information and respect the socio-professional profile of teachers e their understanding and respect for computational thinking. You can conclude that the definition of Computational Thinking is still unknown to most of the research participants, in addition to being associated with the use of computers and other digital technologies in the classroom. The concepts of parallelization, data analysis and data collection, linked to computing, more sessions were used, and mathematics the discipline that most frequently uses the concepts covered.

KeyWords: Basic Education, Computer Education, Computational Thinking, Problem solving. 


\section{Introdução}

A tecnologia tem sido cada vez mais presente e vem se tornando um importante pilar para a sociedade contemporânea. Paralelo ao seu crescimento, está o da capacidade de computar. A computação pode ser definida como uma atividade que visa atender um objetivo claro, beneficiando, precisando ou criando dispositivos computacionais e permitindo a resolução de problemas complexos, repetitivos ou perigosos. "É denominada uma área-meio por ser utilizada como suporte ou ferramenta para a resolução de problemas de outras áreas, muitas delas referenciadas como áreas fim" (DE CARVALHO; LORENA, 2017). Como relatado no Model Curriculum for K-12 Computer Science (2011), a compreensão de conceitos da Ciência da Computação é exigida pela grande maioria das profissões do século XXI, uma vez que todas buscam por soluções de problemas em suas áreas. Nesse contexto, surge a necessidade de outra habilidade (importante e menos compreendida): o pensamento computacional (PC) (Blinkstein, 2008).

Mesmo com alguns indícios implícitos nos trabalhos de Seymour Papert que usa o termo para se referir a como a presença de um computador muda a forma de pensar das pessoas (PAPERT, 1980), o PC ganhou destaque apenas em 2006 pela professora de Ciência da Computação e chefe do Departamento de Ciência da Computação na Universidade de Carnegie Mellon, Pittsburgh (PA), Jeannette Jue Wing. A autora defende que o PC se trata de "uma forma para seres humanos resolverem problemas; não é tentar fazer com que seres humanos pensem como computadores" (WING, 2016).

No cenário internacional, a discussão do PC já é evidente. Na Europa, 15 países já integram no seu currículo da Educação Básica o ensino de Ciência da Computação (CC) (BALANSKAT ET.AL., 2015 apud BOLSINHA, 2017). A Inglaterra substituiu uma disciplina obrigatória de informática pela Computing, fundamentada nos conceitos de CC, Tecnologia da Informação (TI) e Letramento Digital. (UK DEPARTMENT FOR EDUCATION, 2013 apud ALMEIDA; VALENTE, 2019). Na Austrália, desde 2014, o componente curricular de tecnologia está presente no currículo na etapa equivalente a educação infantil e ensino fundamental aqui no Brasil, estando organizado nos temas Design e Tecnologias e Tecnologias Digitais. Este último, aborda o pensamento computacional para projetar e implementar soluções digitais (RAABEE; BRACKMANN, 2018).

Apesar de sua importância, o PC é uma habilidade que não está tão difundida na educação básica brasileira em virtude de diversos desafios enfrentados para o seu ensino. Dentre eles, França e Tedesco (2015) apontam a ausência de um currículo que aborde transversalmente ou através de uma disciplina específica conteúdos de computação, a formação de professores especializados, a definição da melhor estratégia de ensino/aprendizagem e a compreensão sobre a melhor maneira de ensinar os conteúdos. As políticas educacionais existentes nos currículos que regem a educação básica estão restritas à abordagem de letramento e inclusão digital, não sendo encontradas menções do ensino de fundamentos de Computação em nenhum documento oficial (BRACKMANN, Christian P. et al, 2016). A Base Nacional Comum Curricular (BNCC) faz referência ao $\mathrm{PC}$, porém, não traz uma definição precisa sobre o que ela entende do termo nem sobre sua utilização dentro de sua proposta.

Desenvolver habilidades computacionais na Educação Básica se faz necessário em virtude de CC ser importante intelectualmente, por promover múltiplos caminhos 
profissionais futuros e pela possibilidade de desenvolvimento da capacidade de resolver problemas, apoiar e relacionar-se transversalmente com outras ciências, motivando os estudantes (CSTA, 2011).

Considerando a realidade apresentada bem como a relevância do tema proposto, o presente trabalho objetiva investigar, através de uma pesquisa exploratória, o ensino de conceitos e abordagens do pensamento computacional nos anos finais do ensino fundamental ( $6^{\circ}$ ao $9^{\circ}$ ano), tendo como estudo de caso algumas escolas públicas no sertão da Paraíba. Partindo deste, também buscou-se identificar a concepção dos professores a respeito do Pensamento Computacional e mapear quais conceitos ligados ao pensamento computacional são abordados com maior frequência na prática pedagógica bem como as principais disciplinas em que eles são utilizados.

O artigo foi organizado em 5 seções. Na Seção 2 é apresentada a fundamentação teórica a respeito do tema. $\mathrm{Na}$ Seção 3 é descrito o processo metodológico desenvolvimento do trabalho. Na Seção 4 são apresentados os resultados das análises realizadas. A Seção 5 finaliza o artigo descrevendo as principais conclusões, limitações e propostas de trabalhos futuros

\section{O Pensamento Computacional}

A capacidade de encontrar as respostas para os problemas que se fazem presentes no nosso dia a dia é algo inerente às diversas áreas do conhecimento, sendo esta estimulada em diferentes fases da vida escolar do estudante. Apesar disso, vem se verificando a baixa habilidade no processo de resolução de problemas pelos estudantes de diversos níveis de ensino, consequência muitas vezes da falta de alternativas que promovam uma maior reflexão e estímulo para que os problemas sejam resolvidos de maneira mais inovadora (KOSCIANSKI e GLIZT, 2017). É neste contexto que o pensamento computacional (PC) pode contribuir com melhorias.

As primeiras aparições deste termo ocorreram implicitamente nos trabalhos de Seymour Papert (1980), em que ele define o PC como sendo o processo de raciocínio reflexivo a partir não só de um estudo, como também da prática de uma linguagem de programação (BRACKMANN, 2017). Ele desenvolveu uma linguagem de programação denominada LOGO que possibilita a introdução da criação de programas interessantes e permite o desenvolvimento de técnicas de soluções de problemas e capacidades de pensamento divergentes e convergentes (MIRANDA, 1990).

Anos depois, em 2006, o termo PC volta a ser encontrado na literatura, desta vez explicitamente, no artigo Computational Thinking, de Jeannette M. Wing (WING, 2006). Nele, a autora descreve a respeito da definição e da importância de sua utilização das mais variadas áreas do conhecimento. Após várias reformulações, sua definição é atualizada ao escrever que o PC "São os processos de pensamento envolvidos na formulação de um problema e que expressam a sua solução ou soluções eficazmente, de tal forma que uma máquina ou uma pessoa possa realizar" (WING, 2014).

Nunes (2011) afirma que é chamado de Pensamento Computacional "O processo cognitivo utilizado pelos seres humanos para encontrar algoritmos para resolver problemas". Assim, podemos perceber que ele se apresenta como uma forma metodológica de solucionar problemas utilizando como suporte conceitos de computação e pensando na automatização dessas soluções. Ele é dividido em quatro conceitos principais ou pilares: a decomposição, a abstração, o reconhecimento de padrões e o algoritmo. 
A decomposição consiste na divisão do problema em partes menores e mais fáceis de seres resolvidas. O Currículo de Referência em Tecnologia e Computação (2018) destaca a compreensão da prática de análise desses problemas, identificando partes que podem ser separadas e de que formas serão reconstituídas pensando na solução de um problema global. A abstração consiste, dado um conjunto de dados, na classificação e organização de modo que possam ser escolhidos elementos mais relevantes para a resolução do problema. Sua utilização possibilita a criação abstrata do que se quer resolver, sendo a maior dificuldade a escolha do que será compreendido sem perder informações relevantes (BRACKMANN, 2017).

O reconhecimento de padrões busca a identificação de características comuns que são apresentadas não só pelos problemas propostos como também pelas suas soluções para que a resolução seja dada de modo mais eficiente. Liukas (2015, apud BRACKMANN, 2017) conceitua-o como a busca de padrões (elementos iguais ou muito similares) nos problemas apresentados de modo que possam ser encontradas similaridades entre suas características e assim desenvolver de forma eficiente a solução problemas complexo. Quanto mais padrões forem identificados, mais fácil e rápido essas soluções serão construídas. Algoritmo consiste em um conjunto de instruções descritas e organizadas para atingir um determinado objetivo através de diagramas, códigos, pseudocódigos ou expresso por alguma linguagem de programação. Agregando os demais, se trata de um conjunto de instruções claras e necessárias para a solução de um problema. É uma descrição de um processo (que resolve um determinado problema (SBC, 2017)

Barr e Stephenson (2011) identificam os principais aspectos computacionais (conceitos) e capacidades transformando-os em um modelo estruturado que foi construído em uma reunião cujo objetivo era entrar em um consenso sobre o significado do PC no ensino fundamental. Tais elementos estão dispostos abaixo, no quadro 1, que descreve cada um desses nove conceitos:

QUADRO 1: Conceitos que compõem o núcleo do PC segundo Barr e Stephenson.

\begin{tabular}{|c|c|}
\hline Conceitos & Definição em Ciência da Computação \\
\hline Coleta de Dados & Processo de coleta de dados ou informações sobre um problema \\
\hline Análise de Dados & Dar sentido aos dados, encontrar padrões e tirar conclusões. \\
\hline Representação de dados & Representar e organizar dados em gráficos, tabelas, texto ou figuras \\
\hline Abstração & Reduzir a complexidade para definir ideias principais \\
\hline Automação & Usar computadores ou máquinas para realizar tarefas repetitivas \\
\hline Algoritmos & Sequência de passos para resolução de um problema \\
\hline Decomposição & Quebrar um problema em tarefas menores e gerenciáveis \\
\hline Paralelização & Organizar recursos para, simultaneamente, realizar tarefas para \\
& alcançar um objetivo comum; \\
\hline Simulação & Envolve experimentos sendo executados usando modelos. \\
\hline
\end{tabular}

Fonte: Adaptação de Barr e Stephenson (2011). 
A aprendizagem dos conceitos voltados ao PC pode ser estimulada utilizando diversas abordagens. A maioria das propostas está relacionada ao uso de programação, primeira prática que surge quando se debate o tema. Porém, como colocado por Valente (2016), a preocupação atual está além do aprendizado sobre programação. Buscam-se novas estratégias para explorar os conceitos computacionais de modo que novas condições sejam criadas para o desenvolvimento do PC. Além da criação de linhas de códigos, existem também outras abordagens mais conhecidas como o uso da robótica educacional, atividades desplugadas ou simplesmente atividades voltadas à lógica de programação.

$\mathrm{Na} \mathrm{BNCC}$ Podem ser encontrados diversos termos que seguem a linha do PC tanto na competência geral 5 como também nas competências específicas em cada área de conhecimento. Não há uma referência direta ao ensino de conceitos de computação, apenas Tecnologias Digitais como um tema integrador. Existem propostas curriculares alternativas como a da SBC, o componente Tecnologias para a aprendizagem do Currículo Cidade de São Paulo e o Currículo de Referência em Tecnologia e computação proposto pela Sociedade de Inovação para a Educação Brasileira (CIEB).

Entender através deste trabalho como o PC vem sendo abordado nas escolas pesquisadas pode fornecer subsídios para a construção de propostas de utilização dos seus conceitos principalmente de modo alinhado à prática docente e às disciplinas da educação básica, ajudando assim na resolução de problemas das mais diversas áreas.

\section{Metodologia}

A presente pesquisa tem, em relação aos seus objetivos, um caráter exploratório, uma vez que "descreve ou caracteriza a natureza das variáveis que se quer conhecer" (KÖCHE, 2011). Como primeira etapa, realizou-se uma pesquisa de campo, que objetivou a obtenção de informações e/ou conhecimentos sobre um problema, para o qual se procura uma resposta, ou de uma hipótese, que se queira comprovar, ou, ainda, descobrir novos fenômenos ou as relações entre eles. (LAKATOS, 2003, p. 186). Utilizou-se um enfoque quantitativo, uma vez que, como aborda Sampiieri, Collado e Lucio (2013, apud Wazlawick, 2014), na formulação do problema, ele busca a descrição, previsão e explicação, além de dados mensuráveis observáveis.

Como campo de estudo, foram escolhidas as regiões intermediárias de Patos e Sousa-Cajazeiras (que correspondem ao sertão do estado da Paraíba). Participaram da pesquisa, 15 dos 88 municípios aptos. Os participantes foram os docentes de 23 escolas públicas (zona urbana e rural) que lecionam em qualquer disciplina nos anos finais do ensino fundamental, que consistem no período do $6^{\circ}$ ao $9^{\circ}$ ano .

A próxima etapa consistiu na coleta de dados. Como técnica de investigação, adotou-se o questionário. Ele foi disponibilizado para os participantes em duas modalidades: online, construído utilizando a plataforma Google Forms para uma possibilidade de alcance maior; e impresso, para aqueles que não tem facilidade para responder usando alguma ferramenta digital. Ele foi construído dividindo-se em duas seções: a primeira abordou o perfil socioprofissional do respondente; a segunda, buscou entender a concepção dos docentes a respeito do pensamento computacional e a frequência de utilização dos conceitos relacionados utilizando, para isso, uma adaptação das questões utilizadas no trabalho de Mannila el. al. (2014). Para a elaboração das perguntas dispostas na segunda seção do questionário, foram utilizados os nove conceitos ligados ao pensamento computacional, definidos por Barr e Stephenson (2011), objetivando perceber a ocorrência com que cada um é utilizado. 
Para a análise dos dados, foi utilizado como instrumento de auxílio a Plataforma Qlick Sense. Ela facilita a exploração dos dados para que seja possível entender o que está acontecendo. Em relação ao cálculo das porcentagens, nas perguntas que abordam a frequência de uso, foram consideradas respostas positivas na análise, aquelas que informaram usar frequentemente ou com muita frequência. Em relação à concordância, foram consideradas positivas as respostas que concordam total ou parcialmente.

\section{Resultados e Análises}

\subsection{Caracterização do grupo de docentes pesquisado}

A análise está baseada nos dados obtidos pelo questionário aplicado, que obteve uma quantidade de 54 respostas válidas. Foram excluídas três respostas por não se encaixarem no perfil da pesquisa, uma vez que duas destas, lecionam no ensino médio e uma ensinava na educação infantil. Responderam no total 27 homens (50\%) e 27 mulheres $(50 \%)$. Quase metade dos professores (46,3\%) tem mais de 30 anos e $(22,2 \%)$ possuem idade entre os 18 e 24 anos.

Com relação ao tempo de serviço, 22,2\% possuem até 03 anos, $18,5 \%$ entre 03 e 06 anos, $16,7 \%$ entre 06 e 10 anos, $25,9 \%$ entre 10 e 20 anos e 16,7\% acima de 20 anos. Sendo 37 da rede estadual $(68,5 \%)$ e 17 da rede municipal (31,5\%), eles possuem os seguintes graus de formação: 3 docentes possuem bacharelado $(5,56 \%), 51$ possuem licenciatura (94,44\%), 22 têm alguma especialização (40,74\%), 7 possuem mestrado $(12,96 \%)$ e 1 tem o título de doutor (1,85\%).Quanto às disciplinas lecionadas, havia16 professores de matemática, 11 de ciências, 9 de Língua Portuguesa, 9 de História, 6 de Educação Física, 6 de Geografia, 3 de Língua Inglesa, 2 de Arte, 1 de Ensino Religioso, 1 de Química, 1 de Física e 1 de Língua Espanhola.

Em relação aos municípios participantes, $46,7 \%$ deles têm população inferior a 8000 habitantes, segundo o senso do IBGE de 2019. Nestes, estão concentrados 31 dos 54 docentes (equivalente a 57,4\%) que responderam à pesquisa.

\subsection{Concepção dos professores a respeito do Pensamento Computacional}

A respeito do pensamento computacional, segunda parte do questionário, a primeira pergunta foi realizada para identificar se o professor conhecia ou já tinha ouvido falar. As respostas nos mostram que PC ainda é um termo desconhecido para a maioria deles ( 25 professores, o equivalente a 46,3\%), sendo este um número superior a $16(29,6 \%)$ que conhecem e de $13(24,1 \%)$ que talvez conheçam o PC.

$\mathrm{O}$ segundo questionamento foi a respeito do que eles pensavam que significava o PC, podendo escolher mais de uma alternativa. Para a formulação da questão, foram utilizadas definições encontradas na literatura, principalmente nos textos de Wing (2016) e Blikstein (2008). Ao analisarmos as respostas, ficou perceptível que a maioria dos professores acredita que o PC está relacionado ao uso de computadores em sala de aula $(50 \%)$ e/ou ao uso de artefatos como componentes físicos e programas $(33,3 \%)$. É comum encontrarmos pessoas com esta opinião, uma vez que estabelecem a ligação entre computação, computadores e as atividades básicas por eles realizadas. Quando associado à ideia de navegação da internet, envio de e-mails ou pra utilização de processadores de texto, este conceito se torna equivocado e não corresponde ao PC. Como defende Blikstein (2008), "Pensamento computacional é saber usar o computador como um instrumento de aumento do poder cognitivo e operacional humano - em V. $18 \mathrm{~N}^{\mathrm{o}} 1$, julho, 2020 DOI: RENOTE 
outras palavras, usar computadores, e redes de computadores, para aumentar nossa produtividade, inventividade e criatividade."

Ramos e Espadeiro (2014), ao refletirem sobre a proposta do pensamento computacional, reforçam que não se trata apenas de uma literacia digital que se apoia apenas na aquisição de conhecimentos básicos sobre informática e manipulação técnica de equipamentos. "É, antes, uma literacia digital que se funda nos alicerces cognitivos e sociais da sociedade do conhecimento e por isso põe em causa os nossos modos de pensar, agir, as predisposições e atitudes face ao papel da tecnologia na sociedade e na educação." Apesar de a melhoria do ensino não ser garantida apenas com a utilização desses aparatos tecnológicos na sala de aula (computadores e/ou softwares específicos), eles exercem uma grande importância, uma vez que podem ser o meio pelo qual os estudantes encontram alternativas para a solução de problemas complexos (BRACKMANN, 2017).

As definições que ocuparam o $3^{\circ}$ e $4^{\circ}$ lugar respectivamente sobre a definição do $\mathrm{PC}$, foram as que afirmam que ele envolve a resolução de problemas e que é uma habilidade fundamental a todos, como a aritmética, a leitura e a escrita. Elas trazem duas propostas que estão diretamente alinhadas ao contexto real do pensamento computacional. O $3^{\circ}$, com $29,6 \%$, evidencia a importância do tema incluindo o PC no mesmo patamar da aritmética e da leitura. $\mathrm{O} 4^{\circ}$, com $31,5 \%$, traz a definição que está mais alinhada com o que a literatura define, que é a resolução de problemas. Mesmo sem um conhecimento preciso sobre o que realmente é o PC, os participantes da pesquisa já possuem uma noção sobre a sua importância.

Quando buscamos pelas respostas dos docentes que afirmaram conhecer o PC, podemos verificar que a percepção que prevalece ainda é de que se trata do uso de computadores em sala de aula. As duas opiniões mais escolhidas em seguida são que se trata de programação e que é uma habilidade fundamental. Podemos inferir que mesmo afirmando conhecer o tema aqui trabalhado, ainda há uma percepção mais deslocada do núcleo do Pensamento Computacional.

Através dos dados obtidos, pode-se perceber que resolver problemas é capacidade presente na prática de $96,3 \%$ dos professores. Além disso, esta capacidade está diretamente ligada às habilidades estimuladas pelo $\mathrm{PC}$, pois, como argumenta Mestre et. al. (2015), estas envolvem a capacidade de compreensão das situações propostas e de criação de soluções através de modelos matemáticos, científicos ou sociais. A única resposta que afirma não utilizar a resolução de problemas é de um professor de educação física, que afirmou não conhecer pensamento computacional e julga que se trata de habilidades mecânicas e da forma que o computador pensa. Questionados sobre a frequência de utilização desses problemas, 6 afirmaram utilizar com muita frequência $(11,1 \%), 27$ utilizam frequentemente $(50,7 \%), 15$ utilizam ocasionalmente $(27,8 \%), 5$ raramente $(9,3 \%)$ e apenas 1 nunca utiliza $(1,9 \%)$.

É interessante observar que, dos dados coletados, majoritariamente os professores afirmam trabalhar em situação-problema, mas desconhecem PC, ou seja, os professores podem utilizar algumas das bases do PC sem conhecê-lo formalmente.

\subsection{Conceitos alinhados ao PC mais utilizados}

Em relação aos conceitos ligados ao pensamento computacional, considerando a soma das respostas dos professores que usam frequentemente (na maioria das aulas) e com muita frequência (em todas as aulas), podemos verificar, que Paralelização, Análise 
de Dados e Coleta de dados são os três mais utilizados. Eles possuem respectivamente $70.37 \%, 66.67 \%$ e $62.96 \%$ das respostas, considerando todos os professores.

A paralelização está relacionada com a divisão de tarefas em pequenas partes para serem executadas colaborativamente com o objetivo de alcançar um objetivo. A análise de dados consiste no estímulo dos jovens para obterem significado de dados para que eles possam ser transformados em informações ou na solução de um determinado problema. A coleta de dados diz respeito â seleção dos dados mais importantes para se resolver determinada situação.

Seguindo a mesma ideia anterior, os conceitos menos utilizados (consistindo nas respostas raramente e nunca) são a Automação, a Abstração e a Simulação, correspondendo à $27.78 \%, 22.22 \%$ e $18.52 \%$, respectivamente de respostas negativas. A baixa frequência do uso de automação pode estar relacionada com a baixa utilização de ferramentas tecnológicas por indisponibilidade da escola ou falta de conhecimento para manuseio das mesmas. Uma informação não esperada é também a utilização com pouca frequência do conceito de abstração, relacionado com a filtragem de dados de modo que o foco seja dado aos mais relevantes. Isso reduz a complexidade e define a ideia principal do problema.

\section{GRÁFICO 1: Frequência de utilização dos conceitos do PC}

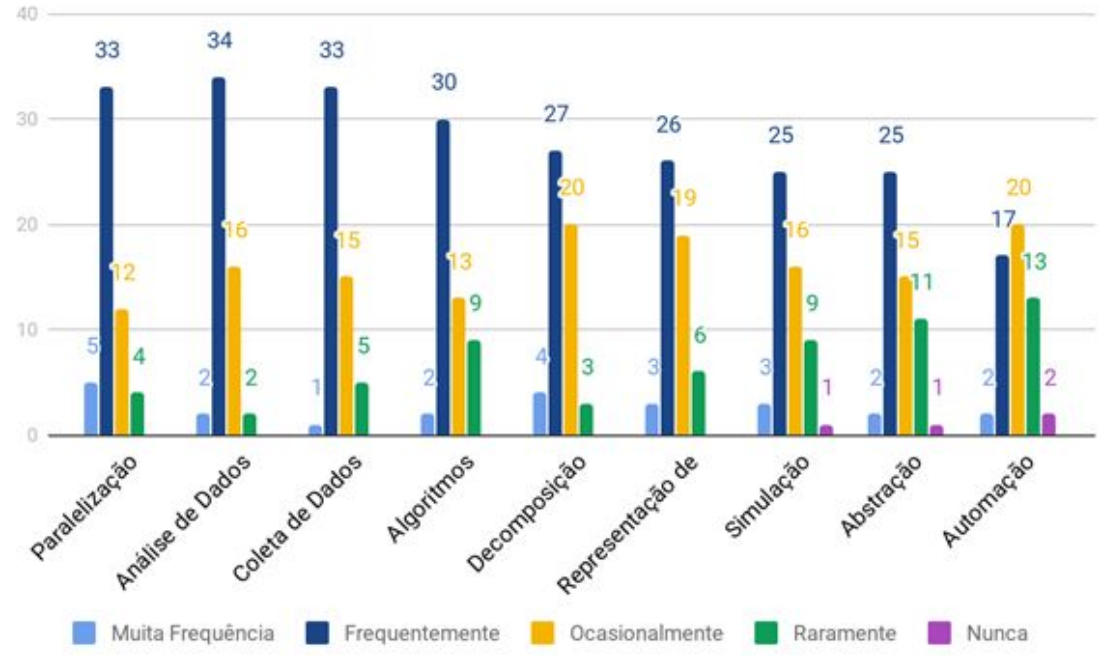

Fonte: Autoria Própria (2020)

Através da análise, pode-se identificar que matemática é a disciplina que mais utiliza conceitos do pensamento computacional. Já era algo esperado por ser uma área de conhecimentos que está diretamente ligada com a resolução de problemas. De acordo com os Parâmetros Curriculares Nacionais (1998, apud MESTRE, 2017), a resolução de problemas contribui para o processo de ensino e aprendizagem da matemática de forma construtivista, uma vez que o aluno é levado à interpretação e à construção de estratégias de solução para situações problema, ponto central da atividade. Além disso, ele coloca que "a resolução de problemas não é, por si só, uma atividade e sim uma orientação para a aprendizagem dos conceitos, procedimentos e atitudes matemáticas.". Dentro da disciplina de matemática, os conceitos que mais são abordados são os de decomposição, a paralelização, a análise de dados e os algoritmos. $\mathrm{Na}$ matemática, eles representam respectivamente a resolução de operações e expressões usando a ordem de precedência, aplicação de ordem de operações em expressões, a capacidade de extrair valores de gráficos, tabelas, etc. para inferir ou V. $18 \mathrm{~N}^{\mathrm{o}} 1$, julho, 2020 RENOTE DOI: 
concluir algo e a sistematização de soluções dos problemas em uma série de passos que devem ser seguidos (BARR; STEPHENSON, 2011)

\section{Considerações Finais}

O presente trabalho buscou, em seu contexto geral, identificar a utilização do Pensamento Computacional através de seus conceitos, tendo como campo de pesquisa cidades do sertão paraibano, hoje identificado como regiões intermediárias Sousa-Cajazeiras e Patos. Nesse contexto, participaram da pesquisa, docentes de 23 escolas divididas entre 15 cidades que lecionam disciplinas nos anos finais do ensino fundamental.

Em relação à percepção dos professores a respeito do $\mathrm{PC}$, com base na análise dos dados apresentada na seção 4.2, podemos concluir que se trata de um termo ainda desconhecido pela maioria. Ainda que alguns conheçam, pode-se perceber que há uma percepção equivocada sobre as suas definições. Existe uma associação considerável entre o PC e o uso de tecnologias digitais como computadores em sala de aula.

Em suas aulas, os professores informaram que abordam com uma grande frequência situações/problemas a serem resolvidos, o que permite a utilização de conceitos de computação para o auxílio da construção das soluções desses problemas. Foram identificados como conceitos mais frequentes, como apresentado na seção 4.3, a paralelização, a análise e a coleta de dados. Como menos frequente, foi identificado a automação, que trabalha exatamente com ferramentas de hardware e software que possam servir de auxílio nesse processo de construção de soluções, que está relacionada com a definição mais popular. Dentre as disciplinas, matemática é a que usa mais recorrentemente os conceitos apresentados. Se trata de algo previsto, por ser uma área que trabalha diretamente com a resolução de problemas.

A pesquisa contribuiu para a identificação de uma realidade presente em diversos cenários no Brasil, principalmente quando se trata de cidades de pequeno porte. Esses resultados podem servir de subsídios para a construção de propostas pautadas na falta de formação dos professores, de uma estrutura mínima adequada e de estratégias mais condizentes com esses dois fatores anteriores.

Como trabalhos futuros, espera-se uma investigação mais profunda a respeito do que os professores entendem sobre cada conceito e sobre como cada um é abordado na prática. Pretende-se também a construção de um plano de estratégias que possam ser aplicadas e posteriormente avaliadas para a adoção do PC em uma cidade da região. É necessário, dentro deste plano, a criação de uma formação continuada para professores do ensino fundamental com técnicas que possam ser implementadas em suas práticas para o estímulo de novas maneiras de se resolver problemas utilizando os conceitos do pensamento computacional.

\section{Referências Bibliográficas}

BARR, Valerie; STEPHENSON, Chris. Bringing computational thinking to K-12: what is Involved and what is the role of the computer science education community?. Inroads, v. 2, n. 1, p. 48-54, 2011.

BLIKSTEIN, P. (2008). O pensamento computacional e a reinvenção do computador na educação. Disponível em: http://www.blikstein.com Acesso em: Janeiro/2020. 
BRACKMANN, C. P. Desenvolvimento do pensamento computacional através de atividades desplugadas na educação básica. Tese (Doutorado) - Universidade do Rio Grande do Sul. Porto Alegre, 2017.

BRACKMANN, Christian P. et al. Pensamento computacional: Panorama nas américas. In: XVIII Simpósio Internacional de Informática Educativa, SIIE. 2016. p. 197.

CSTA, ISTE. Operational definition of computational thinking for K12 education, 2011. Disponível em: $<$ https://id.iste.org/docs/ct-documents/computational-thinking-operational-definition-fly er.pdf?sfvrsn $=2>$.

DE CARVALHO, André CPLF; LORENA, Ana Carolina. Introdução à Computação Hardware, Software e Dados. 2017.

FRANÇA, Rozelma; TEDESCO, Patrícia. Desafios e oportunidades ao ensino do pensamento computacional na educação básica no Brasil. In: Anais dos Workshops do Congresso Brasileiro de Informática na Educação. 2015. p. 1464.

KOSCIANSKI, André; GLIZT, Fabiana Rodrigues de Oliveira. O Pensamento Computacional nos anos iniciais do Ensino Fundamental. Revista Novas Tecnologias na Educação. CINTED-UFRGS, v. 15, n. 2, 2017.

LAKATOS, Eva Maria. Fundamentos de metodologia científica. 5 ed. São Paulo : Atlas 2003

MANNILA, Linda. et al. Computational Thinking in K-9 Education. Proceedings Of The Working Group Reports Of The 2014 On Innovation \& Technology In Computer Science Education Conference - Iticse-wgr '14, [s.1.], p.1-29, 2014. ACM Press. http://dx.doi.org/10.1145/2713609.2713610.

MESTRE, Palloma Alencar Alves et al. O uso do pensamento computacional como estratégia para resolução de problemas matemáticos. 2017.

MIRANDA, Guilhermina Lobato. Linguagem Logo. Análise Psicológica, v. 8, p. 117-120, 1990.

NUNES, D. J. Ciência da Computação na Educação Básica. ADUFRGS - Sindical, 6. jun. 2011. Disponível em: http://adufrgs.org.br/artigos/ciencia-da-computacao-na-edu cacao-basica. Acesso em: 21/01/2019

PAPERT, Seymour. Mindstonns. New York: Basic Rooks, v. 607, 1980.

WAZLAWICK, Raul. Metodologia de pesquisa para ciência da computação. Elsevier Brasil, 2017.

WING, Jeannette M. Computational thinking. Communications of the ACM, v. 49, n. 3, p. 33-35, 2006.

WING, Jeannette M. Computational thinking benefits society. 40th Anniversary Blog of Social Issues in Computing, v. 2014, 2014.

WING, Jeannette M. PENSAMENTO COMPUTACIONAL-Um conjunto de atitudes e habilidades que todos, não só cientistas da computação, ficaram ansiosos para aprender e usar. Revista Brasileira de Ensino de Ciência e Tecnologia, v. 9, n. 2, 2016.

RAMOS, José Luís; ESPADEIRO, Rui Gonçalo. Os futuros professores e os professores do futuro. Os desafios da introdução ao pensamento computacional na escola, no currículo e na aprendizagem. Educação, Formação \& Tecnologias-ISSN 1646-933X, v. 7, n. 2, p. 4-25, 2014. 